\title{
Drug-Induced Osteoporomalacia
}

Bone formation and mineralisation require calcium and phosphate together with active metabolites of vitamin D. Drugs affecting the vitamin D system may cause both osteoporosis and osteomalacia by several mechanisms:

Blockers of Vitamin D Production The elderly and institutionalised individuals with limited nutrition and sunlight exposure are at particular risk.

Inhibitors of Vitamin D Absorption Vitamin $\mathrm{D}$, a fat-soluble vitamin, is absorbed in the jejunum and ileum, in combination with bile acids. Therefore, bile acid-binding resins such as cholestyramine and colestipol interfere with this process and inhibit vitamin $\mathrm{D}$ absorption.

Interference with Vitamin D Metabolism To be active, vitamin $\mathrm{D}$ must first be metabolised in the liver and then in the kidney. Drugs such as anticonvulsants or rifampicin induce drug-metabolising enzymes in the liver which then accelerate the catabolism of vitamin D and its metabolites. Some reports have shown that $20-65 \%$ of patients with epilepsy receiving anticonvulsants such as phenytoin or phenobarbital developed osteoporosis and/or osteomalacia, especially if they were institutionalised. These patients were at an increased risk of fractures during their epileptic seizures. Patients taking these anticonvulsants require higher doses of vitamin $\mathrm{D}$ to achieve a positive calcium balance, with doses up to 4000 IU per day. Anticonvulsants such as sodium valproate do not induce the hepatic drug-metabolising enzymes and therefore have no impact on vitamin D metabolism.

Antagonists of Vitamin D Action Glucocorticoids interfere with intestinal calcium absorption, but they are not direct antagonists of vitamin $\mathrm{D}$ at the receptor level. There are no known drugs that directly interfere with the actions of active vitamin D at the target-tissue level.

Inhibitors of Phosphate Absorption Hypophosphataemia is a major cause of osteomalacia, and the most important drug-induced form is caused by excessive ingestion of aluminiumcontaining antacids, which inhibit intestinal phosphate absorption.

Inhibitors of Bone Mineralisation Aluminiuminduced osteomalacia is mainly found in patients on haemodialysis and on total parenteral nutrition. Etidronate, the first bisphosphonate, also induced disturbances in mineralisation when given in higher doses. However, no reports have been published of osteomalacia caused by nitrogencontaining BP. Bone biopsies of patients treated with alendronate for more than 7 years have shown no evidence of demineralisation. Fluoride in high doses often showed evidence of abnormal mineralisation, and this defect is aggravated by concomitant low calcium and vitamin D intake. 